 \\ TAIRA \\ MOUNTaiNS \\ Mathematical Publications}

DOI: $10.2478 / \mathrm{tmmp}-2014-0009$

Tatra Mt. Math. Publ. 58 (2014), 101-109

\title{
ON SOME MODIFICATION OF ŚWIA̧TKOWSKI PROPERTY
}

\author{
Gertruda Ivanova — ElżBieta Wagner-Bojakowska
}

\begin{abstract}
We introduce some families of functions $f: \mathbb{R} \rightarrow \mathbb{R}$ modifying the Darboux property analogously as it was done by [Maliszewski, A.: On the limits of strong Światkowski functions, Zeszyty Nauk. Politech. Łódź. Mat. 27 (1995), 87-93], replacing continuity with $\mathcal{A}$-continuity, i.e., the continuity with respect to some family $\mathcal{A}$ of subsets in the domain. We prove that if $\mathcal{A}$ has $\left(^{*}\right)$-property then the family $\mathcal{D}_{\mathcal{A}}$ of functions having $\mathcal{A}$-Darboux property is contained and dense in the family $\mathcal{D} \mathcal{Q}$ of Darboux quasi-continuous functions.
\end{abstract}

A function $f: \mathbb{R} \rightarrow \mathbb{R}$ has the intermediate value property if, on each interval $(a, b) \subset \mathbb{R}$, the function $f$ assumes every real value between $f(a)$ and $f(b)$. In 1875, J. Darboux [4] showed that this property is not equivalent to the continuity and every derivative has the intermediate value property.

The intermediate value property is usually called the Darboux property and a function having the intermediate value property is called a Darboux function.

Let $\mathcal{D}$ denote the class of Darboux functions. To simplify our notation, we will write:

$$
<a, b>=(\min \{a, b\}, \max \{a, b\}) .
$$

In 1977, T. M ańk and T. Ś w i a̧ t k ow s ki [16] defined some modification of the Darboux property. They considered a family of functions with the so-called Świątkowski property.

Definition 1 ([16]). A function $f: \mathbb{R} \rightarrow \mathbb{R}$ has Świątkowski property if for each interval $(a, b) \subset \mathbb{R}$ there exists a point $x_{0} \in(a, b)$ such that $f\left(x_{0}\right) \in$ $<f(a), f(b)>$ and $f$ is continuous at $x_{0}$.

In 1995, A. M a lis z e w s k i investigated a class of functions which possesses some stronger property.

Definition 2 ([14]). A function $f: \mathbb{R} \rightarrow \mathbb{R}$ has the strong Świątkowski property if for each interval $(a, b) \subset \mathbb{R}$ and for each $\lambda \in<f(a), f(b)>$ there exists a point $x_{0} \in(a, b)$ such that $f\left(x_{0}\right)=\lambda$ and $f$ is continuous at $x_{0}$.

(C) 2014 Mathematical Institute, Slovak Academy of Sciences.

2010 Mathematics Subject Classification: 26A15, $54 \mathrm{C} 08$.

Keywords: Darboux property, strong Świątkowski property, quasi-continuity. 


\section{GERTRUDA IVANOVA — ELŻBIETA WAGNER-BOJAKOWSKA}

The family of all strong Świątkowski functions will be denoted by $\mathcal{D}_{s}$.

In 2009, Z. Grande considered some modification of strong Świątkowski property replacing the continuity with the approximate continuity.

Definition $3([5])$. A function $f: \mathbb{R} \rightarrow \mathbb{R}$ has the ap-Darboux property if for each interval $(a, b) \subset \mathbb{R}$ and for each $\lambda \in<f(a), f(b)>$ there exists a point $x_{0} \in(a, b)$ such that $f\left(x_{0}\right)=\lambda$ and $f$ is approximately continuous at $x_{0}$.

In the sequel, the family of all functions with the ap-Darboux property will be denoted by $\mathcal{D}_{a p}$.

Let $\mathcal{I}$ be a $\sigma$-ideal of the sets of the first category. In [6] and [7, we introduce a family of functions $f: \mathbb{R} \rightarrow \mathbb{R}$ modifying the Darboux property analogously as it was done by Z. Grande and replacing approximate continuity with $\mathcal{I}$-approximate continuity, i.e., the continuity with respect to $\mathcal{I}$-density topology in the domain (see [3], [19], 20], 23], 24]).

Definition 4. A function $f: \mathbb{R} \rightarrow \mathbb{R}$ has the $\mathcal{I}$-ap-Darboux property if for each interval $(a, b) \subset \mathbb{R}$ and for each $\lambda \in<f(a), f(b)>$ there exists a point $x_{0} \in(a, b)$ such that $f\left(x_{0}\right)=\lambda$ and $f$ is $\mathcal{I}$-approximately continuous at $x_{0}$.

The family of all functions with the $\mathcal{I}$-ap-Darboux property will be denoted by $\mathcal{D}_{\mathcal{I}-a p}$.

Let $\mathcal{A} \subset \mathcal{P}(\mathbb{R})$, where $\mathcal{P}(\mathbb{R})$ is a family of all subsets of $\mathbb{R}$. To simplify our considerations, we need the following definition.

Definition 5. We will say that $f: \mathbb{R} \rightarrow \mathbb{R}$ is $\mathcal{A}$-continuous at a point $x \in \mathbb{R}$ if for each open set $V \subset \mathbb{R}$ with $f(x) \in V$ there exists a set $A \in \mathcal{A}$ such that $x \in A$ and $f(A) \subset V$. We will say that $f: \mathbb{R} \rightarrow \mathbb{R}$ is $\mathcal{A}$-continuous if $f$ is $\mathcal{A}$-continuous at each point $x \in \mathbb{R}$.

It is not difficult to see that if $\mathcal{A}$ is the Euclidean topology $\tau_{e}$, then the notion of the $\mathcal{A}$-continuity is equivalent to the notion of the continuity in the classical sense. If $\mathcal{A}$ is the density topology $\tau_{d}$, then we have the approximate continuity. If $\mathcal{A}$ is the $\mathcal{I}$-density topology $\tau_{\mathcal{I}}$, then we obtain the $\mathcal{I}$-approximate continuity. If $\mathcal{A}$ is some topology $\tau$ on $\mathbb{R}$, then the $\mathcal{A}$-continuity is a continuity between $(\mathbb{R}, \tau)$ and $\left(\mathbb{R}, \tau_{e}\right)$.

Of course, $\mathcal{A}$ need not be a topology. Let $\bar{A}(\operatorname{Int}(A))$ denote the closure (interior) of the set $A$ in the Euclidean topology. A set $A \subset \mathbb{R}$ is said to be semi-open if there is an open set $U$ such that $U \subset A \subset \bar{U}$ (see [11]). It is not difficult to see that $A$ is semi-open if and only if $A \subset \overline{\operatorname{Int}(A)}$. The family of all semi-open sets will be denoted by $\mathcal{S}$. A function $f: \mathbb{R} \rightarrow \mathbb{R}$ is semi-continuous if for each set $V$ open in the Euclidean topology the set $f^{-1}(V)$ is semi-open (see [1]). 


\section{ON SOME MODIFICATION OF ŚWIẠTKOWSKI PROPERTY}

Definition 6. A function $f: \mathbb{R} \rightarrow \mathbb{R}$ is quasi-continuous at a point $x$ if for every neighbourhood $U$ of $x$ and for every neighbourhood $V$ of $f(x)$ there exists a non-empty open set $G \subset U$ such that $f(G) \subset V$. A function $f: \mathbb{R} \rightarrow \mathbb{R}$ is quasi-continuous if it is quasi-continuous at each point.

A. Neubrunnová [18] proved that $f$ is semi-continuous if and only if it is quasi-continuous.

Obviously, $\mathcal{S}$ is not a topology and if $\mathcal{A}$ is the family of semi-open sets $\mathcal{S}$, then the $\mathcal{A}$-continuity is equivalent to the quasi-continuity.

Definition 7. We will say that $f: \mathbb{R} \rightarrow \mathbb{R}$ has $\mathcal{A}$-Darboux property if for each interval $(a, b) \subset \mathbb{R}$ and each $\lambda \in<f(a), f(b)>$ there exists a point $x \in(a, b)$ such that $f(x)=\lambda$ and $f$ is $\mathcal{A}$-continuous at $x$.

The family of all functions having $\mathcal{A}$-Darboux property will be denoted by $\mathcal{D}_{\mathcal{A}}$. It is easy to see that if $\mathcal{A}$ is the Euclidean topology $\tau_{e}$, then $\mathcal{D}_{\mathcal{A}}=\mathcal{D}_{\tau_{e}}=\mathcal{D}_{s}$, if $\mathcal{A}$ is the density topology $\tau_{d}$, then $\mathcal{D}_{\mathcal{A}}=\mathcal{D}_{\tau_{d}}=\mathcal{D}_{\text {ap }}$, and if $\mathcal{A}$ is the $\mathcal{I}$-density topology, then $\mathcal{D}_{\mathcal{A}}=\mathcal{D}_{\tau_{\mathcal{I}}}=\mathcal{D}_{\mathcal{I}-a p}$.

The set $A$ is of the first category at the point $x$ (see [9]) if there exists an open neighbourhood $G$ of $x$ such that $A \cap G$ is of the first category. By $D(A)$ we will denote the set of all points $x$ such that $A$ is not of the first category at $x$.

Let $\mathcal{B} a$ be a family of all functions having the Baire property.

Definition 8. We will say that the family $\mathcal{A}$ has (*)-property if

1. $\tau_{e} \subset \mathcal{A} \subset \mathcal{B} a$;

2. $A \subset D(A)$ for each $A \in \mathcal{A}$.

It is not difficult to see that the wide class of topologies has $(*)$-property, for example, Euclidean topology, $\mathcal{I}$-density topology, topologies constructed in [10] by E. Łazarow, R. A. Johnson and W. Wilczyński or topology constructed by R. Wiertelak in 22. Some families of sets which are not the topologies also have $(*)$-property, for example, the family of semi-open sets, however, it does not have the density topology.

Definition 9. We will say that $f: \mathbb{R} \rightarrow \mathbb{R}$ has q-property if for each $(a, b) \subset \mathbb{R}$ and for each non-empty open interval $(C, D) \subset f((a, b))$ there exists a non-empty open interval $(c, d) \subset(a, b)$ such that $f((c, d)) \subset(C, D)$.

LEMma 1. Darboux function $f: \mathbb{R} \rightarrow \mathbb{R}$ has q-property if and only if $f$ is quasi-continuous.

Proof. $\Rightarrow$ : Let $x \in \mathbb{R}$. There are two cases:

1. $f$ is constant on some neighbourhood of $x$. Then, $f$ is continuous at $\mathrm{x}$. 


\section{GERTRUDA IVANOVA — ELŻBIETA WAGNER-BOJAKOWSKA}

2. $f$ is constant on no neighbourhood of $x$. Let $(a, b)$ and $(p, q)$ be arbitrary intervals such that $x \in(a, b)$ and $f(x) \in(p, q)$. From the Darboux property, $f((a, b))$ is a non-degenerate interval and

$$
f(x) \in(p, q) \cap f((a, b)) .
$$

Hence, $(p, q) \cap f((a, b))$ is a non-degenerate interval and it contains some non-empty interval $(C, D)$. From q-property, there exists a non-empty open interval $(c, d) \subset(a, b)$ such that $f((c, d)) \subset(C, D) \subset(p, q)$.

$\Leftarrow$ : Suppose $f: \mathbb{R} \rightarrow \mathbb{R}$ is quasi-continuous and $(a, b) \subset \mathbb{R}$. Let $(C, D)$ be an arbitrary non-empty open interval contained in $f((a, b))$. Then, there exists a point $x \in(a, b)$ such that $f(x) \in(C, D)$. Hence, from Definition 6 , there exists a non-empty open interval $(c, d) \subset(a, b)$ such that $f((c, d)) \subset(C, D)$.

LEMma 2. If $\mathcal{A}$ has (*)-property and $f \in \mathcal{D}_{\mathcal{A}}$, then $f$ has q-property.

Proof. Let $(a, b)$ be such that $f((a, b))$ is a non-degenerate interval, and let $(C, D) \subset f((a, b))$.

Fix $y \in(C, D)$ and a number $\epsilon>0$ with $[y-\epsilon, y+\epsilon] \subset(C, D)$. Clearly,

$$
[y-\epsilon, y+\epsilon] \subset f((a, b)) \text {. }
$$

Since $f \in \mathcal{D}_{\mathcal{A}}$, there exists a point $x \in(a, b)$ such that $f(x)=y$ and $f$ is $\mathcal{A}$-continuous at $x$. Hence, we can find a set $A_{x} \in \mathcal{A}$ satisfying $x \in A_{x}$ and $f\left(A_{x}\right) \subset(y-\epsilon, y+\epsilon)$.

As $\mathcal{A}$ has $\left(^{*}\right)$-property, $A_{x}$ has the Baire property and is not of the first category at $x$. Hence, $A_{x} \cap(a, b)$ has the Baire property and is not of the first category at $x$, too. Consequently,

$$
A_{x} \cap(a, b)=G \Delta P=(G \backslash P) \cup(P \backslash G),
$$

where $G$ is open and $P$ is of the first category in the Euclidean topology. Obviously, $G \neq \emptyset$, hence, there exists an interval $(c, d) \subset G$. Observe that $(c, d) \backslash P \subset(a, b)$, so, $(c, d) \subset(a, b)$ and $(c, d) \backslash P \subset A_{x}$, i.e., $f((c, d) \backslash P) \subset$ $f\left(A_{x}\right) \subset(y-\epsilon, y+\epsilon)$.

Now, let us prove that $f((c, d)) \subset[y-\epsilon, y+\epsilon]$. Put

$$
V=\mathbb{R} \backslash[y-\epsilon, y+\epsilon] \text {. }
$$

Clearly,

$$
f^{-1}(V) \cap(c, d)=(c, d) \backslash f^{-1}([y-\epsilon, y+\epsilon])
$$

and

$$
(c, d) \backslash P \subset f^{-1}(f((c, d) \backslash P)) \subset f^{-1}([y-\epsilon, y+\epsilon]) .
$$

From (11) and (2), we obtain

$$
f^{-1}(V) \cap(c, d) \subset P .
$$




\section{ON SOME MODIFICATION OF ŚWIẠTKOWSKI PROPERTY}

Assume that $f((c, d)) \cap V \neq \emptyset$, i.e., there exists $x_{1} \in f^{-1}(V) \cap(c, d)$. From (3), we can find a point $x_{2} \in\left(x_{1}, d\right) \backslash f^{-1}(V)$. The set $V$ is open, hence there exists a point $Z \in V \cap<f\left(x_{1}\right), f\left(x_{2}\right)>$. As $f \in \mathcal{D}_{\mathcal{A}}$, we can find a point $z \in\left(x_{1}, x_{2}\right)$ such that $f(z)=Z$ and $f$ is $\mathcal{A}$-continuous at $z$, i.e., there exists a set $A_{z} \in \mathcal{A}$ such that $z \in A_{z}$ and

$$
f\left(A_{z}\right) \subset V
$$

By (3) and (4), we have

$$
A_{z} \cap\left(x_{1}, x_{2}\right) \subset f^{-1}(V) \cap(c, d) \subset P,
$$

i.e., $z \in A_{z} \cap\left(x_{1}, x_{2}\right)$, and $A_{z}$ is of the first category at $z$, which is a contradiction, as $\mathcal{A}$ has (*)-property and $A_{z} \in \mathcal{A}$. Hence, $f((c, d)) \cap V=\emptyset$, i.e., $f((c, d)) \subset$ $[y-\epsilon, y+\epsilon] \subset(C, D)$.

From now on, by $\mathcal{Q}$ we will denote the family of quasi-continuous functions and by $\mathcal{D} \mathcal{Q}$ the family of quasi-continuous functions with the Darboux property.

Theorem 1. If $\mathcal{A}$ has (*)-property, then $\mathcal{D}_{s} \subset \mathcal{D}_{\mathcal{A}} \subset \mathcal{D} \mathcal{Q}$.

P r o o f. It follows easily from Lemma 1 and Lemma 2.

Let us show that these inclusions may be proper for different families $\mathcal{A}$.

Lemma 3. There exists a family $\mathcal{A} \neq \tau_{e}$ with $\left({ }^{*}\right)$-property such that $\mathcal{D}_{s}=\mathcal{D}_{\mathcal{A}}$.

Proof. Let $\mathcal{A}=\tau_{h}$, where $\tau_{h}=\left\{G \backslash P: G \in \tau_{e}\right.$ and $\left.P \in \mathcal{I}\right\}$ is a H. Has himot o topology (see [8]). Obviously, $\tau_{e} \subset \tau_{h}$, so $\mathcal{D}_{s} \subset \mathcal{D}_{\tau_{h}}$.

Let us show that $\mathcal{D}_{\tau_{h}} \subset \mathcal{D}_{s}$. Fix a function $f \in \mathcal{D}_{\tau_{h}}$, an interval $(a, b)$ such that $f(a) \neq f(b)$ and $Z \in<f(a), f(b)>$. Hence, as $f$ has the $\tau_{h}$-Darboux property, there exists a point $z \in(a, b)$ such that $f(z)=Z$ and $f$ is $\tau_{h}$-continuous at $z$.

Let us observe that $f$ is $\tau_{e}$-continuous at $z$. Indeed, fix $\epsilon>0$. Hence, there exists a set $A \in \tau_{h}$ such that $z \in A$ and

$$
f(A) \subset\left(f(z)-\frac{\epsilon}{2}, f(z)+\frac{\epsilon}{2}\right) .
$$

The set $A$ is open in $\tau_{h}$, therefore $A=G \backslash P$, where $G$ is open and $P$ is of the first category. Obviously $A \subset G$, so $z \in G$, and there exists $\delta>0$ such that $(z-\delta, z+\delta) \subset G$. Hence $(z-\delta, z+\delta) \backslash P \subset G \backslash P=A$, so

$$
f((z-\delta, z+\delta) \backslash P) \subset\left(f(z)-\frac{\epsilon}{2}, f(z)+\frac{\epsilon}{2}\right) .
$$

Analogously as in the proof of Lemma 2 (replacing $(c, d)$ with $(z-\delta, z+\delta)$ and $(y-\epsilon, y+\epsilon)$ with $[f(z)-\epsilon / 2, f(z)+\epsilon / 2])$ we obtain that

$$
f((z-\delta, z+\delta)) \subset\left[f(z)-\frac{\epsilon}{2}, f(z)+\frac{\epsilon}{2}\right] .
$$




\section{GERTRUDA IVANOVA — ELŻBIETA WAGNER-BOJAKOWSKA}

Consequently,

$$
f((z-\delta, z+\delta)) \subset(f(z)-\epsilon, f(z)+\epsilon),
$$

so $f$ is $\tau_{e}$-continuous at $z$ and $f \in \mathcal{D}_{s}$.

We will say that the sets of the form $\bigcup_{n=1}^{\infty}\left(a_{n}, b_{n}\right)$ or $\bigcup_{n=1}^{\infty}\left[a_{n}, b_{n}\right]$ are right-hand interval sets (left-hand interval sets) at $x \in \mathbb{R}$ if $b_{n+1}<a_{n}<b_{n}\left(b_{n}<\right.$ $\left.a_{n+1}<b_{n+1}\right)$ for $n \in \mathbb{N}$ and $\lim _{n \rightarrow \infty} a_{n}=x$.

LEMma 4. There exists a family $\mathcal{A} \subset \mathcal{P}(\mathbb{R})$ with (*)-property such that $\mathcal{D}_{s} \subsetneq$ $\mathcal{D}_{\mathcal{A}} \subsetneq \mathcal{D} \mathcal{Q}$.

Proof. Let $\mathcal{A}=\tau_{\mathcal{I}}$, where $\tau_{\mathcal{I}}$ denotes the $\mathcal{I}$-density topology. Then, $\mathcal{A}$ has (*)-property, so $\mathcal{D}_{s} \subset \mathcal{D}_{\mathcal{A}} \subset \mathcal{D} \mathcal{Q}$.

Let us prove $\mathcal{D}_{s} \subsetneq \mathcal{D}_{\tau_{\mathcal{I}}} \subsetneq \mathcal{D} \mathcal{Q}$.

Assume that $A=\bigcup_{n=1}^{\infty}\left(a_{n}, b_{n}\right)$ is a right interval set at zero and put

$$
f_{A}(x)= \begin{cases}1-x & \text { for } x \leq 0, \\ 1-\frac{1}{n} & \text { for } x \in\left[a_{n}, b_{n}\right], n \in \mathbb{N} \\ 0 & \text { for } x=\frac{a_{n}+b_{n+1}}{2}, n \in \mathbb{N} \text { and for } x \in\left[b_{1}, \infty\right) \\ \text { linear } & \text { on the intervals }\left[b_{n+1}, \frac{a_{n}+b_{n+1}}{2}\right],\left[\frac{a_{n}+b_{n+1}}{2}, a_{n}\right], n \in \mathbb{N}\end{cases}
$$

Obviously, $f_{A}$ is continuous at each point $x \in \mathbb{R}, x \neq 0$, and assumes value 1 only at 0 . Hence, $f_{A} \notin \mathcal{D}_{s}$. On the other hand, it is easy to see, that $f_{A} \in \mathcal{D} \mathcal{Q}$ for each right-hand interval set $A$.

Let $B$ be a right-hand interval set at 0 such that 0 is a right-hand $\mathcal{I}$-density point of $B$ (see [6]). Obviously, $f_{B} \in \mathcal{D}_{\tau_{\mathcal{I}}} \backslash \mathcal{D}_{s}$.

Let $C$ be a right-hand interval set at 0 such that 0 is a right-hand $\mathcal{I}$-dispersion point of $C$. Then, $f_{C}$ is not $\mathcal{I}$-approximately continuous at 0 (see [6] for more details) and assumes value 1 only at point 0 , so $f_{C} \in \mathcal{D} \mathcal{Q} \backslash \mathcal{D}_{\tau_{\mathcal{I}}}$.

Lemma 5. There exists a family $\mathcal{A} \subset \mathcal{P}(\mathbb{R})$ with $\left({ }^{*}\right)$-property such that $\mathcal{D}_{\mathcal{A}}=\mathcal{D} \mathcal{Q}$.

P r o of. Let us observe that the family $\mathcal{S}$ of semi-open sets has (*)-property. Fix $A \in \mathcal{S}$. As $A \subset \overline{\operatorname{Int}(A)}$, the set $A$ is a union of two sets, where the first is open and the second is nowhere dense, so $A$ has the Baire property. Fix a point $x \in A$ and an interval $(a, b)$ such that $x \in(a, b)$. Hence $x \in \overline{\operatorname{Int}(A)}$, so $(a, b) \cap \operatorname{Int}(A) \neq \emptyset$, i.e., $(a, b) \cap A$ is of the second category and $x \in D(A)$. Consequently, $A \subset D(A)$.

By Theorem 1] we have $\mathcal{D}_{\mathcal{S}} \subset \mathcal{D} \mathcal{Q}$.

Let $f \in \mathcal{D} \mathcal{Q}$. Fix $(a, b) \subset \mathbb{R}$ such that $f(a) \neq f(b)$, and let $Z \in<f(a), f(b)>$. From the Darboux property, there exists $z \in(a, b)$ such that $f(z)=Z$. As $f$ is quasi-continuous at $z, f \in \mathcal{D}_{\mathcal{S}}$.

Consequently, we can put $\mathcal{A}=\mathcal{S}$. 


\section{ON SOME MODIFICATION OF ŚWIATTKOWSKI PROPERTY}

Let $\mathcal{U}$ be a family of all functions such that for each $a<b$ and for each set $A \subset[a, b]$ with $\operatorname{card}(A)<\operatorname{card}(\mathbb{R})$, the set $f([a, b] \backslash A)$ is dense in $\langle f(a), f(b)\rangle$. Let $\mathcal{Q U}=\mathcal{Q} \cap \mathcal{U}$. As $\mathcal{D} \subset \mathcal{U}$ (for more details, see [2]), we have $\mathcal{D} \mathcal{Q} \subset \mathcal{Q U}$.

A function $f: \mathbb{R} \rightarrow \mathbb{R}$ is cliquish at the point $x$ if for every neighbourhood $U$ of $x$ and for each $\epsilon>0$ there exists a non-empty open set $G \subset U$ such that $|f(y)-f(z)|<\epsilon$ for each $y, z \in G$. A function $f: \mathbb{R} \rightarrow \mathbb{R}$ is cliquish if it is cliquish at each point.

It is noted (see [15]) that each quasi-continuous function is cliquish and a function $f$ is cliquish if and only if the set of discontinuity points of $f$ is of the first category. Hence the sum of two cliquish functions is cliquish.

Let Cliq denote the family of all cliquish functions.

From the Lind en ba u m's Theorem ([12]), it is well-known that an arbitrary function can be represented as a sum of two Darboux functions (see also [1, Chapter I, Theorem 4.1]). In [5], Z. Gr a n d e proved that every real function $f$ defined on some interval is the sum of two functions from $\mathcal{D}_{a p}$.

W.S i e r p iński in 21] proved that an arbitrary function can be represented as a limit of a pointwise convergent sequence of Darboux functions. Z. Grande showed the analogous result for his family, i.e., that every real function $f$ defined on some interval is the limit of a pointwise convergent sequence of functions from $\mathcal{D}_{a p}$. As for each family $\mathcal{A}$ having (*)-property $\mathcal{D}_{\mathcal{A}} \subset \mathcal{D} \mathcal{Q}$ it is easily seen that analogous results for our family do not hold, moreover:

TheOREM 2. If $\mathcal{A}$ has (*)-property, then:

1. $f \in \mathcal{D}_{\mathcal{A}}+\mathcal{D}_{\mathcal{A}}$ if and only if $f \in \mathcal{D}_{s}+\mathcal{D}_{s}$ if and only if $f \in \mathcal{D} \mathcal{Q}+\mathcal{D} \mathcal{Q}$ if and only if $f \in \mathcal{C}$ liq;

2. $f$ is cliquish if and only if $f$ is a limit of pointwise convergent sequence of functions having $\mathcal{A}$-Darboux property;

3. $f \in \mathcal{Q U}$ if and only if $f$ is a limit of uniformly convergent sequence of functions having $\mathcal{A}$-Darboux property.

Pr o of. Let $\mathcal{A}$ be an arbitrary family having (*)-property.

1. From Theorem 1 we obtain $\mathcal{D}_{s} \subset \mathcal{D}_{\mathcal{A}} \subset \mathcal{D} \mathcal{Q} \subset \mathcal{C}$ liq. Consequently, $\mathcal{D}_{s}+\mathcal{D}_{s} \subset$ $\mathcal{D}_{\mathcal{A}}+\mathcal{D}_{\mathcal{A}} \subset \mathcal{D} \mathcal{Q}+\mathcal{D} \mathcal{Q} \subset \mathcal{C}$ liq $+\mathcal{C}$ liq $=\mathcal{C}$ liq. On the other hand, by [13, Corollary 3.4, Chapter II], we have $\mathcal{C}$ liq $\subset \mathcal{D}_{s}+\mathcal{D}_{s}$, so $\mathcal{C l i q}=\mathcal{D}_{s}+\mathcal{D}_{s}=$ $\mathcal{D}_{\mathcal{A}}+\mathcal{D}_{\mathcal{A}}=\mathcal{D} \mathcal{Q}+\mathcal{D} \mathcal{Q}$.

2. By [14, Corollary 6], the function $f$ is cliquish if and only if there exists a pointwise convergent sequence of Darboux quasi-continuous functions $\left\{f_{n}\right\}_{n \in \mathbb{N}}$ such that $f=\lim _{n \rightarrow \infty} f_{n}$, and if and only if there exists a pointwise convergent sequence of strong Świątkowski functions $\left\{g_{n}\right\}_{n \in \mathbb{N}}$ such 


\section{GERTRUDA IVANOVA — ELŻBIETA WAGNER-BOJAKOWSKA}

that $f=\lim _{n \rightarrow \infty} g_{n}$. So, by Theorem 1, we have that $f$ is a limit of pointwise convergent sequence of functions having $\mathcal{A}$-Darboux property if and only if $f$ is cliquish.

3. In [17, Theorem 4], the author proved that $f \in \mathcal{Q U}$ if and only if $f$ is a limit of uniformly convergent sequence of Darboux quasi-continuous functions. On the other hand, by [14, Corollary 5], $f \in \mathcal{Q U}$ if and only if $f$ is a limit of uniformly convergent sequence of strong Świątkowski functions. So, by Theorem $1, f \in \mathcal{D} \mathcal{Q}$ if and only if $f$ is a limit of uniformly convergent sequence of functions having $\mathcal{A}$-Darboux property.

Let us introduce a metric $\rho$ in the space $\mathcal{U} \mathcal{Q}$ in the following way:

$$
\rho(f, g)=\min \{1, \sup \{|f(t)-g(t)|: t \in \mathbb{R}\}\} .
$$

Corollary 1. If $\mathcal{A}$ has $\left(^{*}\right)$-property, then $\mathcal{D}_{\mathcal{A}}$ is dense in $(\mathcal{D} \mathcal{Q}, \rho)$, and the closure of $\mathcal{D}_{\mathcal{A}}$ equals $\mathcal{Q U}$.

P r o o f. Let $\mathcal{A}$ be a family having (*)-property. By the previous theorem, $f$ is a limit of uniformly convergent sequence of functions having $\mathcal{A}$-Darboux property if and only if $f \in \mathcal{Q U}$, so $\mathcal{D}_{\mathcal{A}}$ is dense in $(\mathcal{Q U}, \rho)$, and the closure of $\mathcal{D}_{\mathcal{A}}$ equals $\mathcal{Q U}$. As $\mathcal{D} \mathcal{Q} \subset \mathcal{Q U}, \mathcal{D}_{\mathcal{A}}$ is dense in $(\mathcal{D} \mathcal{Q}, \rho)$.

Clearly, if $\mathcal{A}_{1} \subset \mathcal{A}_{2}$ then $\mathcal{D}_{\mathcal{A}_{1}} \subset \mathcal{D}_{\mathcal{A}_{2}}$. The opposite implication does not hold: there exist uncomparable families $\mathcal{A}_{1}$ and $\mathcal{A}_{2}$ such that $\mathcal{D}_{\mathcal{A}_{1}} \subset \mathcal{D}_{\mathcal{A}_{2}}$. For example, if $\mathcal{A}_{1}$ is Hashimoto topology and $\mathcal{A}_{2}$ is a family of! semi-open sets, then, by Lemmas 3 and [5] we have $\mathcal{D}_{\mathcal{A}_{1}}=\mathcal{D}_{s} \subsetneq \mathcal{D} \mathcal{Q}=\mathcal{D}_{\mathcal{A}_{2}}$. Simultaneously, if $P$ is a set of the first category dense in $\mathbb{R}$, then $\mathbb{R} \backslash P$ is open in the Hashimoto topology but not semi-open. On the other hand, $[0,1]$ is semi-open but not open in the Hashimoto topology.

\section{REFERENCES}

[1] BRUCKNER, A. M.: Differentiation of Real Functions, in: Lecture Notes in Math., Vol. 659, Springer, Berlin, 1978.

[2] BRUCKNER, A. M.-CEDER, J. G.-WEISS, M. L.: Uniform limits of Darboux functions, Colloq. Math. 15 (1966), 65-77.

[3] CIESIELSKI, K.-LARSON, L.-OSTASZEWSKI, K.: I-density continuous functions, Mem. Amer. Math. Soc. 515 (1994), 133 p.

[4] DARBOUX, G.: Memoire sur les fonctions discontinues, Ann. Sci. Scuola Norm. Sup. 4 (1875), 57-112.

[5] GRANDE, Z.: On a subclass of the family of Darboux functions, Colloq. Math. 117 (2009), 95-104. 


\title{
ON SOME MODIFICATION OF ŚWIẠTKOWSKI PROPERTY
}

[6] IVANOVA, G.: Remarks on some modification of the Darboux property, Bull. Soc. Sci. Lettres Łódź Sér. Rech. Déform. 72 (2014), 91-100.

[7] IVANOVA, G.-WAGNER-BOJAKOWSKA, E.: On some modification of Darboux property, Math. Slovaca (to appear).

[8] HASHIMOTO, H.: On the *-topology and its application, Fund. Math. 91 (1976), 5-10.

[9] KURATOWSKI, A.-MOSTOWSKI, A.: Teoria Mnogości Wraz ze Wstȩpem do Opisowej Teorii Mnogości. PWN, Warszawa, 1978.

[10] ŁAZAROW, E.-JOHNSON, R. A.-WILCZYŃSKI, W.: Topologies related to sets having the Baire property, Demonstratio Math. 21 (1989), 179-191.

[11] LEVINE, N.: Semi-open sets and semi-continuity in topological spaces, Amer. Math. Monthly 70 (1963), 36-41.

[12] LINDENBAUM, A.: Sur quelques propriétés des fonctions de variable réelle, Ann. Soc. Math. Polon. 6 (1927), 129-130.

[13] MAlisZeWSKI, A.: Darboux Property and Quasi-Continuity. A uniform approach. WSP, Słupsk, 1996.

[14] MALISZEWSKI, A.: On the limits of strong Światkowski functions, Zeszyty Nauk. Politech. dz. Mat. 27 (1995), 87-93.

[15] MALISZEWSKI, A.: Sums and products of quasi-continuous functions, Real Anal. Exchange 21 (1995-96), 320-329.

[16] MAŃK, T.-ŚWIA̧TKOWSKI, T.: On some class of functions with Darboux's characteristic, Zeszyty Nauk. Politech. dz. Mat. 11 (301) (1977), 5-10.

[17] NATKANIEC, T.: On quasi-continuous functions having Darboux property, Math. Pannon. 3 (1992), 81-96.

[18] NEUBRUNNOVÁ, A.: On certain generalizations of the notion of continuity, Matematický časopis 23 (1973), 374-380.

[19] POREDA, W.-WAGNER-BOJAKOWSKA, E.-WILCZYŃSKI, W.: A category analogue of the density topology, Fund. Math. 125 (1985), 167-173.

[20] POREDA, W.-WAGNER-BOJAKOWSKA, E.-WILCZYŃSKI, W.: Remarks on $\mathcal{I}$-density and $\mathcal{I}$-approximately continuous functions, Comm. Math. Univ. Carolinae 26 (1985), 553-563.

[21] SIERPIŃSKI, W.: Sur une propriété de fonctions réelles quelconques définies dans les espaces métriques, Matematiche (Catania) 8 (1953), 73-78.

[22] WIERTELAK, R.: A generalization of density topology with respect to category, Real Anal. Exchange 32 (2006/2007), 273-286.

[23] WILCZYŃSKI, W.: A generalization of the density topology, Real Anal. Exchange 8 (1982-83), 16-20.

[24] WILCZYŃSKI, W.: A category analogue of the density topology, approximate continuity and the approximate derivative, Real Anal. Exchange 10 (1984-85), 241-265.

\author{
Gertruda Ivanova \\ Elżbieta Wagner-Bojakowska \\ University of Eódź \\ Faculty of Mathematics and \\ Computer Science \\ ul. Banacha 22 \\ PL-90-238 Eódź \\ POLAND \\ E-mail: gertruda@math.uni.lodz.pl \\ wagner@math.uni.lodz.pl
}

